Received: 2017.02.20 Accepted: 2017.05.31 Published: 2017.09.14

\title{
Angiomyolipoma of the Adrenal Gland: A Report of Two Cases and Review of the Literature
}

Authors' Contribution: Study Design A Data Collection B Statistical Analysis C Data Interpretation D Manuscript Preparation E Literature Search F Funds Collection G

\author{
ABCDEFG Obin Ghimire \\ ABCDG Li Wenzheng \\ $A B C D$ Liu Huaping \\ BDF \\ CDE \\ DE
}

Department of Radiology, Xiangya Hospital, Central South University, Changsha, Hunan, P.R. China
Corresponding Author: Conflict of interest:

\begin{abstract}
Case series
Patient:

Final Diagnosis:

Symptoms:

Medication:

Clinical Procedure:

Specialty:

Objective:

Background:

Case Reports:

Conclusions:

Male, 36 - Female, 61

Adrenal angiomyolipoma

Asymptomatic

$-$

Radiology

\section{Rare disease}

Angiomyolipoma is a benign tumor composed of blood vessels, smooth muscle cells, and adipose tissue and has been described as belonging to the group of tumors of perivascular epithelioid cell origin (PEComa), commonly found in the kidneys and strongly associated with tuberous sclerosis. Only a few cases of extra-renal angiomyolipoma have previously been reported in the literature, most commonly in the liver. Adrenal angiomyolipoma is very rare, is usually asymptomatic, and is often found incidentally, with only 14 previously reported cases identified in the literature.

We report two cases of adrenal angiomyolipoma that were identified by abdominal computed tomography (CT). The first case presented in a 36-year-old man and was an oval-shaped adrenal mass, measuring $5.2 \times 4.2 \times 3.1 \mathrm{~cm}$. The second case presented in a 61 -year-old woman and was a round-shaped mass measuring $8.6 \times 9.5 \times 8.1 \mathrm{~cm}$. Both patients underwent adrenalectomy. Histopathology and immunohistochemistry confirmed the diagnosis of benign angiomyolipoma composed of adipose tissues, blood vessels, and smooth muscle cells.

We present two rare cases of adrenal angiomyolipoma. We have reviewed the literature and identified 14 other cases of adrenal angiomyolipoma, and discuss the clinical, radiological, and pathological features of this rare tumor.
\end{abstract}

MeSH Keywords:

Full-text PDF:

\section{Adrenal Gland Neoplasms • Angiomyolipoma • Retroperitoneal Neoplasms}

https://www.amjcaserep.com/abstract/index/idArt/903908

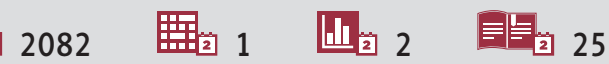




\section{Background}

Angiomyolipoma is a benign tumor of mesenchymal origin, composed of smooth muscle cells, small vessels or vascular channels, and adipose tissue, which confirming the diagnosis [1]. Renal angiomyolipoma was first described by Morgan in 1951 [2].

Angiomyolipoma is now believed to belong to the group of tumors of perivascular epithelioid cell origin (PEComa), commonly found in the kidneys, and strongly associated with tuberous sclerosis. Approximately $50 \%$ of cases of angiomyolipoma are associated with tuberous sclerosis and when this association is present, angiomyolipoma is more likely to be multiple and bilateral [3]. Extrarenal angiomyolipoma is very rare; the liver is the most common extrarenal site [4]. To our knowledge, only 14 previous cases of adrenal angiomyolipoma have been reported previously in the literature in the English language (Table 1). We present the findings of two cases of adrenal angiomyolipoma diagnosed and treated at our hospital.

\section{Case Reports}

\section{Case 1}

A 36-year-old man was referred to our hospital due to a mass in right upper abdomen detected on ultrasonography (US). A year before this referral, the patient underwent a routine medical checkup at his local hospital, where a small mass was detected in the right upper abdomen. The patient was advised at that time for follow-up within a year. At one-year followup, a repeat abdominal US showed an increase in the size of the mass. Therefore, the patient was referred to our hospital for further evaluation and management.
On presentation to our hospital, the patient had no symptoms. On physical examination, there was no palpable mass, no tenderness, and the patient was completely asymptomatic. His medical and surgical history were unremarkable.

The patient was referred for non-enhanced computed tomography (CT) scan of the abdomen, which showed a well-defined, oval-shaped mass, with fat attenuation, -114 Hounsfield units $(\mathrm{HU})$, in the right suprarenal area, measuring $5.2 \times 4.2 \times 3.1 \mathrm{~cm}$. Following contrast-enhanced CT imaging, the mass was nonenhancing, with a mean $\mathrm{CT}$ value of $-58 \mathrm{HU}$ in the arterial phase and $-38 \mathrm{HU}$ in the venous phase. A small blood vessel was seen inside the mass that enhanced more in the venous phase with a mean CT value of $+168 \mathrm{HU}$ (Figure 1A). Some non-enhancing scattered lesions were seen within the adrenal mass. Based on the CT imaging findings, a provisional diagnosis of adrenal angiomyolipoma was made.

Laboratory findings, including serum biochemistry and circulating tumor markers were normal. Due to the increase in the size of the mass during the previous year, the patient was advised to have the mass surgically removed.

At surgery, a soft, yellow, fatty tumor arising from the right adrenal gland with no capsule was found. Laparoscopic right adrenalectomy was performed with no postoperative complications. Histopathology of the excised tumor showed adipose tissue that contained abnormally formed small blood vessels, and smooth muscle cells (Figure 1B). No hematopoietic tissues were noted within the tumor. Immunohistochemistry was performed using a panel of antibodies to tumor markers, which showed that the tumor cells were positive for smooth muscle actin (SMA), CD34, and Melan-A. The cell proliferation marker,
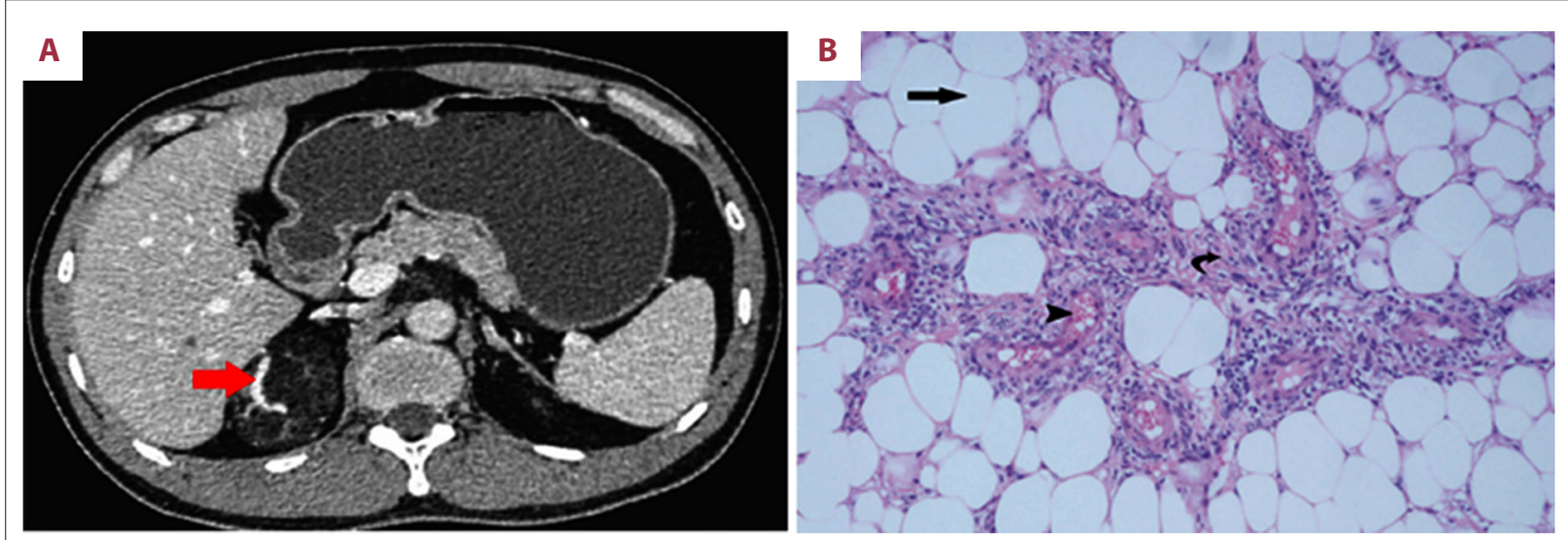

Figure 1. Case 1. (A) Contrast-enhanced axial computed tomography (CT) scan of the abdomen showing a well-defined, oval-shaped, hypodense mass in the right adrenal gland containing contrast-enhancing blood vessels (red arrow). (B) Photomicrograph of the histology of the angiomyolipoma showing a mixture of adipose tissue (black straight arrow), smooth muscle cells (black curved arrow), and blood vessels (black arrow head). 


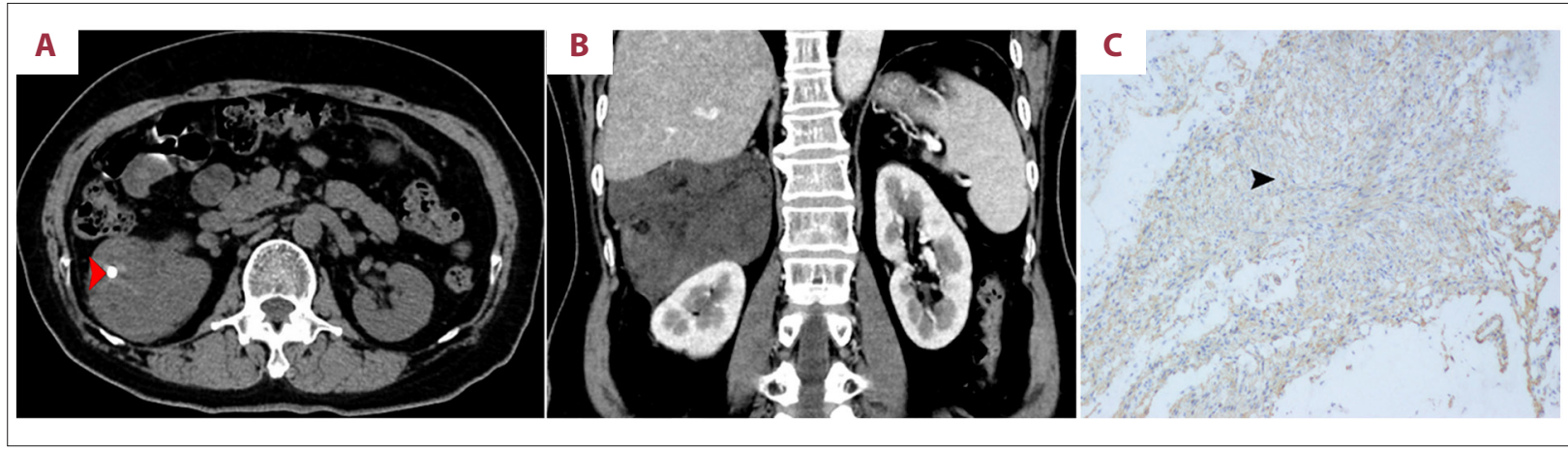

Figure 2. Case 2. (A) Non-enhanced axial computed tomography (CT) scan of the abdomen showing a right retroperitoneal mass with a small area of calcification (red arrow head). (B) Contrast-enhanced coronal CT scan of the abdomen showing a large, welldefined, right suprarenal mass with minimal enhancement and a few area of adipose tissue. (C) Photomicrograph of the light microscopy of the immunohistochemical localization of smooth muscle actin (SMA) shows brown immunostaining localized to spindle-shaped smooth muscle cells (black arrow head).

Ki67 showed a cell proliferation rate of $<5 \%$. These findings confirmed a diagnosis of a benign angiomyolipoma.

\section{Case 2}

A 61-year-old woman was referred to our hospital following detection of an abnormal right upper abdominal mass on US during a routine health checkup at her local hospital. On attending our hospital, she denied any pain or change in bowel and bladder habit. However, she mentioned having a sense of a mass in the right upper quadrant for the previous few days. The patient had a history of hypertension and diabetes mellitus.

On physical examination of her abdomen, a firm, mobile, nontender mass was palpable in right upper quadrant. The patient was advised to have a CT scan of the abdomen, which showed a well-defined, round mass with a clear and smooth margin in the right retroperitoneum measuring $8.6 \times 9.5 \times 8.1 \mathrm{~cm}$ with mixed density of soft tissue, adipose tissue ( $+91-49 \mathrm{HU})$ and a small area of calcification (+1728 HU) (Figure 2A). After contrast administration, there was minimal enhancement on CT in some areas of the tumor, but most of the mass was nonenhancing; +98-61 HU in the arterial phase and +104-84 HU in the venous phase (Figure 2B). Laboratory findings, including serum biochemistry and circulating tumor markers were normal. Based on the imaging findings, a differential diagnosis of the mass was made that included gastrointestinal stromal tumor (GIST), teratoma, dysgerminoma, and lipomatous adrenal tumor.

The patient underwent open laparotomy, and a soft tissue mass within the right adrenal gland was seen with clear margins. Regional lymph nodes were not enlarged. The mass was successfully excised, and the patient's postoperative course was uneventful.
Histopathology of the excised tumor showed adipose tissue that contained abnormally formed small blood vessels, and smooth muscle cells exhibiting positive immunoreactivity for SMA (Figure 2C), desmin, CD34, and HHF35 (also for smooth muscle actin), supporting a diagnosis of right adrenal angiomyolipoma.

\section{Discussion}

Table 1 summarizes the clinical data of from the previously published 14 case reports of adrenal angiomyolipoma and the two cases we have presented in this case report. As the current two cases have shown, the presence of all three components, vascular, smooth muscle cell, and adipose tissue, are required to make a histological diagnosis of angiomyolipoma, as first described by Morgan in 1951 [1]. Reported cases of angiomyolipoma arising from the kidney have shown that about $50 \%$ of are associated with tuberous sclerosis and may be bilateral or multiple [3]. These associations were not found in our two cases of adrenal angiomyolipoma.

Angiomyolipoma is now believed to belong to the group of tumors of perivascular epithelioid cell origin (PEComa) [3]. It has also been reported that angiomyolipoma has some similarities with lymphangioleiomyomatosis (LAM) [7]. Extra-renal angiomyolipoma is very rare, and the liver is the most common site [4].

Adrenal angiomyolipoma is very rare. Including our two cases, to best of our knowledge, only 16 cases of adrenal angiomyolipoma have now been reported (Table 1). The mean age of presentation is 47.6 years, with six cases in men and ten cases in women. Interestingly, 11 out of 16 cases were noted to be on the right side (Table 1). Venyo et al. reviewed and summarized the findings of extrarenal retroperitoneal angiomyolipoma and 
Table 1. Clinical data of cases of adrenal angiomyolipoma from the literature review.

\begin{tabular}{|c|c|c|c|c|c|}
\hline Pt. No. & Reference & Age/Sex (yrs.) & Presentation & Side & Size $(\mathrm{cm}) / /$ maging procedures \\
\hline 1. & Lam et al., 2001[1] & $46 / F$ & Incidental finding & $\mathrm{L}$ & $8 \mathrm{~cm}$ diameter $(\mathrm{CT})$ \\
\hline 2.* & Lam et al., 2001 [1] & $20 / M$ & $\begin{array}{l}\text { Incidental finding at } \\
\text { nephrectomy }\end{array}$ & L & $\begin{array}{l}0.2 \mathrm{~cm} \text { diameter on nephrectomy } \\
\text { specimen }(\mathrm{Sm})\end{array}$ \\
\hline 3. & Li et al., 2015 [4] & $53 / M$ & L upper abdominal pain & $\mathrm{L}$ & $9 \times 6$ (US) \\
\hline 4. & Godara et al., 2007 [5] & $45 / F$ & Epigastric discomfort & $\mathrm{L}$ & $15 \times 12$ (US) \\
\hline 5. & Hu et al., 2012 [6] & $55 / F$ & $\mathrm{R}$ upper abdominal pain & $R$ & $15 \times 16$ (US) (Lg) \\
\hline $6 .^{* *}$ & Sutter et al., 2007 [7] & $32 / F$ & Diffuse abdominal pain & $\mathrm{R}$ & $\begin{array}{l}6 \mathrm{~cm} \text { in maximum transverse } \\
\text { diameter }(\mathrm{CT})\end{array}$ \\
\hline 7. & Gupta et al., 2011 [8] & $42 / M$ & Upper abdominal pain & $\mathrm{R}$ & $8 \times 5.5 \times 4.5(\mathrm{CT})$ \\
\hline 8. & Goswami et al., 2014 [9] & $43 / F$ & $\mathrm{R}$ loin pain & $\mathrm{R}$ & $9.5 \times 8 \times 2(M R I)$ \\
\hline 9. & Yener et al., 2011 [10] & $45 / F$ & R subcostal pain & $\mathrm{R}$ & $5 \times 6$ (US) \\
\hline 10. & Monowarul et al., 2012 [11] & $37 / M$ & $\begin{array}{l}\text { Generalized jerking } \\
\text { discomfort and weakness }\end{array}$ & $\mathrm{R}$ & $5.4 \times 4.5$ (US) \\
\hline $11 .^{*}$ & Elsayes et al., 2005 [12] & $49 / F$ & Referred for MRI & R & $12.2 \times 9.8 \times 6.8(\mathrm{MRI})$ \\
\hline 12. & Chee et al., 2010 [13] & $61 / M$ & L loin pain & L & $10 \times 10 \times 10.1(\mathrm{CT})$ \\
\hline 13. & Hafeez et al., 2013 [14] & $72 / F$ & $\mathrm{R}$ upper quadrant pain & $\mathrm{R}$ & $9 \times 8.9(\mathrm{CT})$ \\
\hline 14. & Kwazneski et al., 2016 [15] & $65 / F$ & $\mathrm{R}$ upper abdominal pain & $\mathrm{R}$ & $11.3 \times 9.4$ (US) \\
\hline 15. & PC 1 & $36 / M$ & Incidental finding & $\mathrm{R}$ & $5.2 \times 4.2 \times 3.1(\mathrm{CT})$ \\
\hline 16. & PC 2 & $61 / F$ & Incidental finding & $R$ & $8.6 \times 9.5 \times 8.1(\mathrm{CT})$ \\
\hline
\end{tabular}

${ }^{*}$ Associated with tuberous sclerosis; ${ }^{* *}$ associated with lymphangioleiomyomatosis. PC - present case; $F$ - female; $M$ - male;

L - left; R - right; US - ultrasound; CT - computed tomography; MRI - magnetic resonance imaging; Lg - largest; Sm - smallest.

found that $84 \%$ of patients were women and $16 \%$ were men, which indicates that extra-renal angiomyolipoma predominantly occurs in women [16].

The clinical diagnosis of adrenal angiomyolipoma may be difficult to make. Patients with adrenal angiomyolipoma may present with non-specific symptoms, such as abdominal pain, while most are asymptomatic. As our cases have demonstrated, angiomyolipoma is usually detected as an incidental finding during ultrasound (US) or computed tomography (CT) for an unrelated condition or in a routine medical examination.

The reported size of the adrenal angiomyolipoma varies from $0.2 \mathrm{~cm}$ in diameter to $15 \times 16 \mathrm{~cm}$. Initially, US imaging may be helpful in detecting adrenal angiomyolipoma, but imaging studies such as CT and magnetic resonance imaging (MRI) play an important role in diagnosis. However, due to the diverse morphology of angiomyolipoma on imaging, it is difficult to confirm the diagnosis, and if the tumor is large and heterogeneous, imaging may give a false impression of malignancy.
Also, because it is a vascular tumor, adrenal angiomyolipoma can present with hemorrhage $[13,15]$. Because of its proximity to the kidney, hemorrhage associated with adrenal angiomyolipoma may be misdiagnosed as a primary renal neoplasm or perinephric hematoma [17]. One of the previously reported cases showed intense uptake of fluorine-18-fluorodeoxyglucose (FDG) in positron emission tomography (PET)/ $\mathrm{CT}$ by adrenal angiomyolipoma, which gave a false impression of malignancy [4].

Adrenal angiomyolipoma may also present with central calcification and compression of the inferior vena cava [14]. Scattered calcification on imaging of angiomyolipoma has been initially mistaken for adrenal carcinoma [15]. A highly vascularized adrenal mass supplied by a hypertrophic right inferior suprarenal artery in digital subtraction angiography (DSA) was also reported in the literature in a case of adrenal angiomyolipoma [7]. Bright signal intensity on non-fat-suppressed images with dropout signal on fat-suppressed images using MRI can suggest the presence of a fatty component 
of angiomyolipoma [12]. However, fat is a non-specific component of several other tumors [18].

In Case 1, we noted a small branch of a blood vessel inside the mass of the tumor which might have been suggestive of angiomyolipoma. Schieda et al. have shown that the presence of vessel supplying the tumor in imaging studies may help to differentiate angiomyolipoma from retroperitoneal sarcoma, especially in tumors of larger size [19]. According to our review of previously reported cases, including our two cases of adrenal angiomyolipoma, most of these tumors have shown a well-defined, non-enhancing, non-homogenous mass with clear and smooth margins with low density on CT scan. The presence of fat components together with soft tissue components and a feeding vessel may help in differentiating angiomyolipoma from other tumors, such as myelolipoma, liposarcoma, leiomyoma, and leiomyosarcoma.

When any adrenal mass is detected, even if the patient is symptomatic or the tumor is greater than $6 \mathrm{~cm}$ in size, surgery is required as the risk of malignancy increases with size [20]. Also, large angiomyolipomas may recur locally [5]. Angiomyolipoma has a tendency to rupture spontaneously, and retroperitoneal hemorrhage could occur due to lack the fragility of the abnormal blood vessels in the tumor, which can lack elastin in their walls [15]. Because the risk of spontaneous bleeding increases with size, surgery or selective arterial embolization has been recommended for the treatment of larger angiomyolipoma [3].

In our review of previously published cases of adrenal angiomyolipoma, and including our two cases (Table 1), we noted that all 16 reported cases of adrenal angiomyolipoma were managed surgically. Whenever possible, laparoscopic adrenalectomy should be considered as an initial choice of treatment over open surgery, as it is minimally invasive and carries lower postoperative complications.

Histopathology is required to make the definitive diagnosis of angiomyolipoma, which will contain adipocytes, smooth muscle cells, and blood vessels, with peripherally compressed adrenal cortical tissue. However, in some cases, angiomyolipoma may contain a predominance of with smooth muscle. Although several studies have demonstrated that the precursor cell of angiomyolipoma may have phenotypic homology with the pericyte, the cell of origin remains unknown [21]. In our Case 1 , immunohistochemistry of the tumor tissue confirmed positive immunostaining for SMA, CD34, and Melan-A; in Case 2, immunohistochemistry of the tumor tissue confirmed positive immunostaining for SMA, desmin, CD34, and HHF35. In 2013 , an immunohistochemical study of renal angiomyolipoma reported that all cases were positive for HMB-45 [22]. However, Melan-A was positive in $85 \%$, and SMA was positive in $73 \%$ out of 15 cases studied [22]. Lin et al. reported a case of angiomyolipoma that was negative for HMB-45, Melan-A, and cytokeratin, but positive for CD34 and HHF35 [23]. Desminpositive angiomyolipoma has also been reported in the literature [24]. The cell proliferation marker, Ki-67 is an important marker to consider in the assessment of tumors. In our Case $1, \mathrm{Ki}-67$ showed $<5 \%$ of proliferating cells in the tumor. $\mathrm{Xu}$ et al. mentioned that increased expression of Ki-67 in angiomyolipoma may indicate a diagnosis of epithelioid angiomyolipoma, which carries a less benign prognosis [25]. The presence of multinucleated giant cells in angiomyolipoma has been reported in the literature, which may also be mistaken for malignancy [13]. Angiomyolipoma may present with some increased cellular mitotic activity, but this should not be mistaken for sarcoma [15]. All 16 cases of adrenal angiomyolipoma we have reviewed and summarized (Table 1) were benign.

\section{Conclusions}

Adrenal angiomyolipoma is extremely rare, benign tumor, usually detected incidentally by radiologists during imaging for unrelated conditions. Adrenal angiomyolipoma must be carefully differentiated from other retroperitoneal tumors as it could increase the risk of overdiagnosis, especially if a large tumor is present. Although rare, adrenal angiomyolipoma should be considered as differential diagnosis in adrenal tumors that shows a well-defined, non-enhancing, non-homogenous mass with clear and smooth margins with low density on CT scan. The presence of a fat component together with a soft tissue component and feeding vessel on imaging may help to differentiate angiomyolipoma from other tumors. Histopathology will confirm the diagnosis of angiomyolipoma if the tumor contains adipocytes, smooth muscle cells, and blood vessels. Because health-screening programs that use imaging are increasing around the world, in future, we believe that more cases of adrenal angiomyolipoma will be reported in the literature.

\section{Acknowledgements}

The authors would like to thank Professor Wen Guang Liu from the Department of Pathology, Xiangya Hospital, Central South University for providing assistance.

\section{Conflict of Interest}

None. 


\section{References:}

1. Lam KY, Lo CY: Adrenal lipomatous tumours: A 30 year clinicopathological experience at a single institution. J Clin Pathol, 2001; 54: 707-12

2. Martignoni G, Pea M, Reghellin D et al: PEComas: The past, the present and the future. Virchows Arch, 2008; 452: 119-32

3. Eble JN: Angiomyolipoma of kidney. Seminars Diag Pathol, 1998; 15: 21-40

4. Li W, Pang H, Cao Y et al: High 18F-fluorodeoxyglucose uptake in adrenal angiomyolipoma: Case report and review of literature. Medicine, 2015; 94: e900

5. Godara R, Vashist MG, Singla SL et al: Adrenal angiomyolipoma: A rare entity. Indian J Urol, 2007; 23: 319-20.

6. Hu H, Xi X: Giant adrenal angiomyolipoma. J Clin Endocrinol Metab, 2012; 97: 3835-36

7. Sutter R, Boehler A, Willmann JK: Adrenal angiomyolipoma in lymphangioleiomyomatosis. Eur Radiol, 2007; 17: 565-66

8. Gupta P, Guleria S: Adrenal angiomyolipoma: A case report and review of literature. Res J Med Sci, 2011; 5: 243-46

9. Goswami A, Sharma A, Khullar R et al: Adrenal angiomyolipoma: A case report and review of literature. J Min Access Surg, 2014; 10: 213-15

10. Yener O, Ozcelik A: Angiomyolipoma of the right adrenal gland. ISRN Surg, 2011; 2011: 102743

11. Monowarul I, Amanullah ATM, Alam AKMK: Asymtomatic angiomyolipoma of the right adrenal gland. J Surgical Sci, 2012; 16: 47-48

12. Elsayes KM, Narra VR, Lewis JS Jr., Brown JJ: Magnetic resonance imaging of adrenal angiomyolipoma. J Comput Assist Tomogr, 2005; 29: 80-82

13. Chee Kong $\mathrm{CH}$, Mohamed Rose I, Singam P et al: Angiomyolipoma of the adrenal gland: A case report. Iran Red Crescent Med J, 2010; 12: 489-91

14. Hafeez Bhatti AB, Dar FS, Pervez M: Adrenal angiomyolipoma. J Coll Physicians Surgeons Pakistan, 2013; 23: 663-64
15. Kwazneski D, Merrill M, Young J, Sell H Jr.: Angiomyolipoma and malignant PEComa: Discussion of two rare adrenal tumors. Case Rep Oncol Med, 2016; 2016: 5204092

16. Venyo AK: A Review of the literature on extrarenal retroperitoneal angiomyolipoma. Int J Surg Oncol, 2016; 2016: 6347136

17. Hussain T, Al-Hamali S: Pathophysiology and management aspects of adrenal angiomyolipomas. Ann Royal Coll Surg (Engl), 2012; 94: 224-26

18. Tseng CA, Pan YS, Su YC et al: Extrarenal retroperitoneal angiomyolipoma: Case report and review of the literature. Abdom Imaging, 2004; 29: 721-23

19. Schieda N, Kielar AZ, Al Dandan O et al: Ten uncommon and unusual variants of renal angiomyolipoma: Radiologic-pathologic correlation. Clin Radiol, 2015; 70: 206-20

20. Grumbach MM, Biller BM, Braunstein GD et al: Management of the clinically inapparent adrenal mass ("incidentaloma"). Ann Intern Med, 2003; 138: 424-29

21. D'Antonio A, Caleo A, Caleo 0 et al: Monotypic epithelioid angiomyolipoma of the adrenal gland: An unusual site for a rare extrarenal tumor. Ann Diagn Pathol, 2009; 13: 347-50

22. Esheba Gel S, Esheba Nel S: Angiomyolipoma of the kidney: Clinicopathological and immunohistochemical study. J Egyptian Nat Cancer Inst, 2013; 25: 125-34

23. Lin CY, Tsai CC, Kau HC et al: HMB-45 negative angiomyolipoma of the orbit: A case report and review of the literature. BMC Ophthalmol, 2016; 16: 8

24. L'Hostis H, Deminiere C, Ferriere JM, Coindre JM: Renal angiomyolipoma: A clinicopathologic, immunohistochemical, and follow-up study of 46 cases. Am J Surg Pathol, 1999; 23: 1011-20

25. Xu C, Jiang XZ, Zhao HF et al: The applicability of Ki-67 marker for renal epithelioid angiomyolipoma: Experience of ten cases from a single center. Neoplasma, 2013; 60: 209-14 